\title{
Experimental study of ground vibrations induced by Brussels IC/IR trains in their neighbourhood
}

\author{
Georges Kouroussis ${ }^{\mathrm{a}}$, Calogero Conti and Olivier Verlinden
}

Université de Mons - UMONS, Faculty of Engineering, Department of Theoretical Mechanics, Dynamics and Vibrations, Place du Parc 20, 7000 Mons, Belgium

\author{
Received 30 November 2012, Accepted 11 April 2013
}

\begin{abstract}
In this paper, railway-induced ground vibrations are analysed experimentally with the aim of addressing questions raising from the planning of a new RER network, available in the near future in order to alleviate pollution and the traffic jams. Free field ground vibrations are measured during the passing of InterCity and InterRegion trains. Various sites along the main line L161 Brussels-Luxembourg are chosen and investigated, with specific differences about the track and soil configurations. In order to compare these results with numerical ones, a deep dynamic characterisation of the track, the soil and the train is first performed. The analysis of horizontal and vertical ground vibrations measured during the passage of domestic trains (AM96, AM86, AM80, AM75, HLE27/M4 or M5) at various speeds (from 40 to $120 \mathrm{~km} \cdot \mathrm{h}^{-1}$ ) is then presented. The results show that the ground vibration amplitude depends on various factors: soil configuration, train type and speed, direction of measurement, track quality. In a particular case, a local defect (rail joint) induces large deformations of the soil.
\end{abstract}

Key words: Ground vibration / RER network / peak particle velocity / trains / dynamic soil characteristics

\section{Introduction}

Problems related to vibrations in buildings are a nonnegligible environmental issue in the network design, especially for nearby structures in dense cities. The growing traffic volume, the higher population density and the diminishing distance between the vehicle and the structure amplify the overall vibration nuisance. Although most of general traffic exposure vibrations (smooth surface) are uncomfortable for people, the passing of heavy vehicles on road or track with an uneven surface is often pointed out as the main vibratory nuisance felt by the dwellers.

Brussels, the capital of Belgium and of the European Union, constitutes a heavily urban territory with a few more than one million of residents. The railway network in the Brussels Capital Region $\left(160 \mathrm{~km}^{2}\right)$ consists not only of the urban tramway network but also of the intercity and international train lines, since it represents a vital link in the HST network in the northwest of Europe. Although railway transport appears as the most promising solution to traffic congestion, the development of new lines is confronted to the availability of convenient areas and the mistrust of the dwellers likely to be submitted to new nuisances. These difficulties

\footnotetext{
a Corresponding author:

georges.kouroussis@umons.ac . be
}

have been largely encountered during the implementation of the new RER network. According to Alias [1], the ground vibrations can be categorized in three frequency bands: the low-frequencies (until $15 \mathrm{~Hz}$ ) due to the vehicle dynamics and well transmitted in the ground, the mid-frequencies (from $15 \mathrm{~Hz}$ to $150 \mathrm{~Hz}$ ), due to the track flexibility, and the high-frequencies (over $150 \mathrm{~Hz}$ ), constituting essentially the rolling noise due to the wheel/rail sliding (at these frequencies, the soil strongly absorbs the vibrations). Several attempts have been made to study and quantify the effects and models are useful to analyse the traffic-induced ground vibrations and to reduce their propagation in the soil.

As for other disciplines of engineering, ground vibrations induced by railway traffic are more and more studied by simulation. However, as validation can only be performed by measurements, there is still a need in experimental results. Such results, available in the literature, are often dedicated to a particular field, for example the high-speed case [2-5]. The particularity of these data is that they correspond to a very good track quality, mainly focusing on the (quasi-)static deflection. Dynamic deflection, caused by the track unevenness and the vehicle/track dynamics, can then be neglected. Most of the prediction models are therefore derived from this hypothesis (see for example [6-8]). 


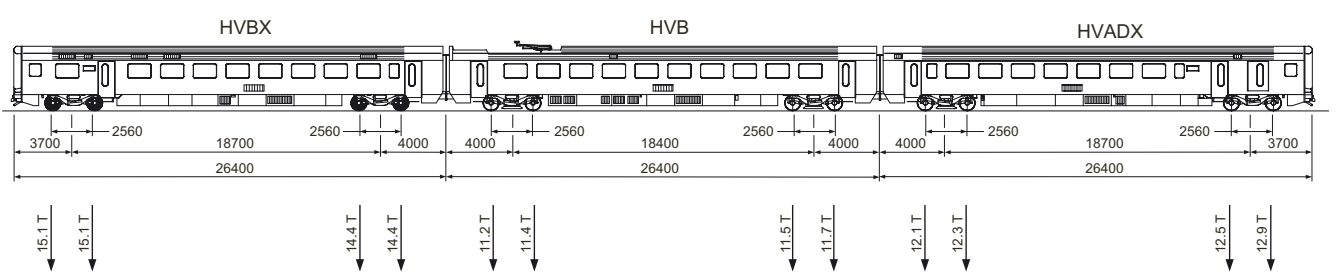

Fig. 1. Configuration of the AM96 electric multiple unit (3-carriages version).

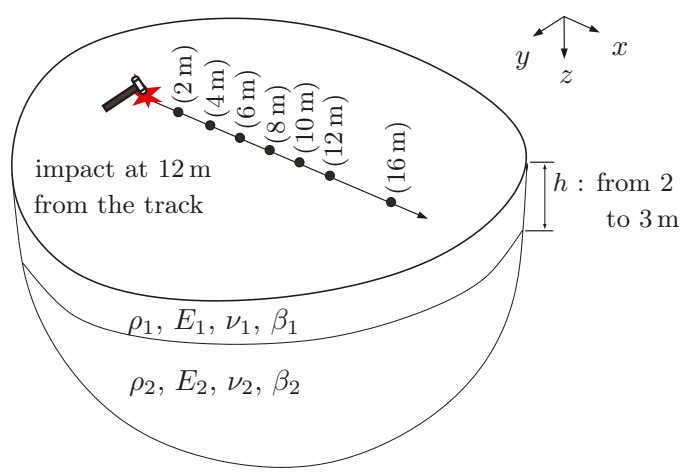

\begin{tabular}{|l|c|c|}
\hline Layer & $\mathbf{1}$ & $\mathbf{2}$ \\
\hline Young's modulus $E$ & $120 \mathrm{MN} / \mathrm{m}^{2}$ & $500 \mathrm{MN} / \mathrm{m}^{2}$ \\
Density $\rho$ & $1600 \mathrm{~kg} / \mathrm{m}^{3}$ & $2500 \mathrm{~kg} / \mathrm{m}^{3}$ \\
Poisson's ratio $\nu$ & 0.3 & 0.3 \\
Velocity $c_{P}$ & $320 \mathrm{~m} / \mathrm{s}$ & $520 \mathrm{~m} / \mathrm{s}$ \\
Velocity $c_{S}$ & $171 \mathrm{~m} / \mathrm{s}$ & $278 \mathrm{~m} / \mathrm{s}$ \\
Viscous damping $\beta$ & $0.0004 \mathrm{~s}$ & $0.0004 \mathrm{~s}$ \\
\hline
\end{tabular}

Fig. 2. Summary of the dynamic and geometrical soil characteristics at site 1 of Watermael.

Before the expansion of the L161 between Watermael and Ottignies in the Brussels region, in situ measurements have been performed. Three sites, located in Watermael (sites 1 and 2) and Ottignies, have been investigated. Ground surface vibrations have been recorded during the passage of Intercity (IC) and InterRegion (IR) domestic trains. The speed $v_{0}$ of the train varied in a large scale, from 40 to $120 \mathrm{~km} \cdot \mathrm{h}^{-1}$. The first intention of these tests was to determine experimentally the vibratory level dependence on train types, track and soil configuration. The second purpose was to obtain a large database in order to validate a prediction scheme, recently developed [9] and validated in the case of tramway [10] and high-speed traffic [11].

The objective of this paper is to present a synthesis of the collected measurements. The train characteristics are briefly reviewed, focusing especially on the AM96 unit, largely used by the Belgian Railway Operator SNCB. In situ soil and track tests are also described, in order to obtain dynamic parameters of these subsystems. The experimental results are finally presented. A particular attention is paid to the impact of the train and the soil configuration, as well as the direction of measurement.

\section{The L161 line between Brussels and Luxembourg}

The L161 is the first section of the line BrusselsLuxembourg (the other being the L162 line). With its $62 \mathrm{~km}$ and two tracks, it constitutes the main railway line in Walloon Region. As part of the development of the new RER network, the L161 line must be expanded with two supplementary tracks. Presently, the speed is limited to $130 \mathrm{~km} \cdot \mathrm{h}^{-1}$. The following descriptions are related to the investigated line before its modification.

\subsection{Domestic trains circulating on the L161}

Various trains circulate on the L161 line, the main ones being:

- the electric multiple unit AM75 acquired at the beginning of 1970 by the SNCB and used for the InterRegion connection (maximal speed: $140 \mathrm{~km} . \mathrm{h}^{-1}$; axle loads: 220 tonnes for a quadruple car);

- the AM80 unit, more recent, developed by the $\mathrm{BN} / \mathrm{ACEC}$ consortium and characterized by a maximal speed of $160 \mathrm{~km} \cdot \mathrm{h}^{-1}$ and a load of 170 tonnes for a three-car configuration;

- the AM86 unit, performing good acceleration for local traffic;

- the AM96 unit, the most recent in the carriage stock and define the major of long-ride trains.

The last unit is essentially reserved to the InterCity connection, due to its excellent passenger comfort. It uses the latest bogie technology from the high speed sector in order to obtain a smoother ride. The AM96 is suitable for speeds up to $160 \mathrm{~km} \cdot \mathrm{h}^{-1}$. Figure 1 summarizes the geometrical parameters of this vehicle. Other vehicles have been investigated during the test, as M4 and M5 coaches, pulled by a HLE27 locomotive.

\subsection{Dynamic soil characteristics}

A lithological description of the site reveals that the soil is composed essentially of a layer of sand of Brussels and a layer of sand and clay of Ypres (Fig. 2), alluvium and bank material as top layers are close dynamic parameters of the first layer. Non-destructive in situ tests have been performed in the three sites in order to determine the dynamic parameters of each soil layer. Mass 


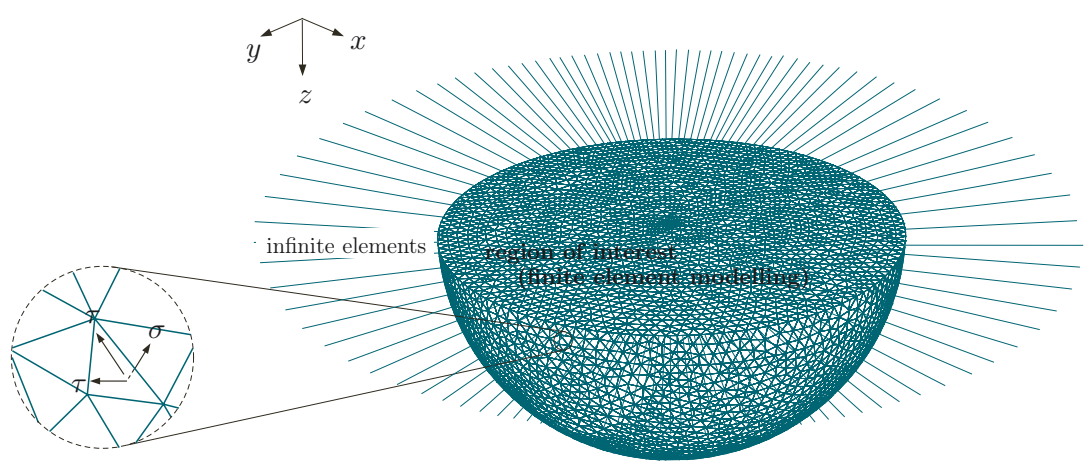

Fig. 3. Finite element model with combined viscous boundary conditions/infinite elements.

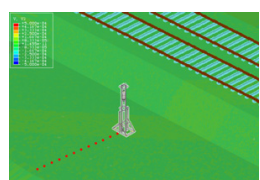

(a) $t=0 \mathrm{~s}$

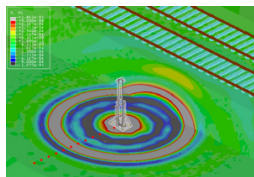

(c) $t=0.10 \mathrm{~s}$

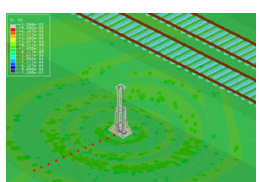

(b) $t=0.05 \mathrm{~s}$

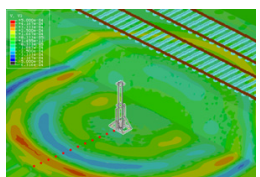

(d) $t=0.15 \mathrm{~s}$

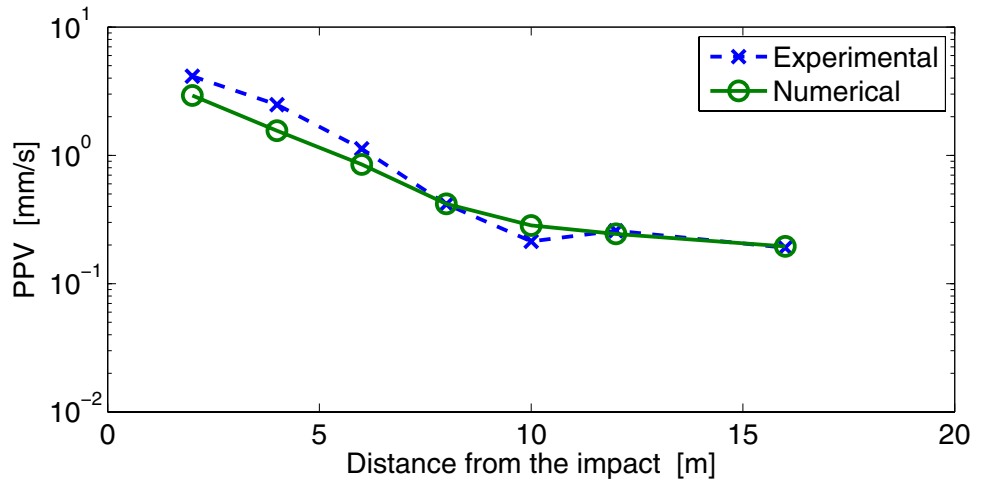

(e) Peak-particle velocity in function of the distance

Fig. 4. Results obtained for the viscous damping evaluation (Watermael site 1).

falling has been performed, each impact being applied on a square foundation $(0.3 \mathrm{~m} \times 0.3 \mathrm{~m})$ from a height of $1 \mathrm{~m}$. Several seismic sensors (Sensor SM-6 low-frequency and Geospace GS-20DM geophones mounted on steel) have been placed at various distances from the impact in order to measure the first arrival time of body waves. Seismic refraction test has been conducted in order to calculate the velocity $c_{\mathrm{P}}$ of primary waves and the local geometry (depth and slope) of each refractor. Associated to other tests (cone penetration test and panda test), the complete values are obtained, as presented in Figure 2 for the site 1 of Watermael. In this case, the first layer has a depth varying from 2 to $3 \mathrm{~m}$ on a distance of $30 \mathrm{~m}$.

Due to the inability of the seismic method to determine the internal damping of the soil, a numerical model has been used in order to update the soil layer damping values by minimizing the gap between experimental $h^{e}(t)$ and numerical $h^{n}(t)$ impulse responses. The model consists of a numerical finite/infinite element (FIEM), based on our recent works [12], which consider efficient nonreflecting boundaries combining viscous elements and infinite elements (Fig. 3). The soil FIEM model takes into account the complex geometry of the site (bank material, inclined layers). Figure 4 shows the peak particle velocity $P P V$ related to the Watermael site 1 for a viscous damping $\beta=0.0004 \mathrm{~s}$ for each identified layer. This approach gives an interesting alternative to estimate the soil damping parameters. Similar results have been obtained for the other sites (Watermael site 2 and Ottignies).

\subsection{Dynamic analysis of the track}

On each site, the track is classically ballasted, composed of concrete sleepers (Watermael sites) or concrete bi-bloc (Ottignies site). Typical UIC 60 is used for rails with a mass $m_{r}$ of $60 \mathrm{~kg} \cdot \mathrm{m}^{-1}$ and a moment of inertia $I_{r}=0.03055 \mathrm{~cm}^{4}$. Concrete mono-bloc sleepers have a mass $m$ of $300 \mathrm{~kg}$ (200 kg for bi-bloc configuration) and a spacing $L$ where the rail is fixed with Pandroll fastening system. During the test, only the track of the first site was investigated. Experimental analysis was performed by measuring the vertical acceleration of the rail and the sleepers induced by a sledge hammer excitation on the foot of the rail, on top of the sleeper. Once the measurements are performed, the vertical experimental receptance can be calculated and compared to numerical receptances, calculated from a finite element model of the track (Fig. 5), according to a three-layer configuration (rail railpad - sleeper - ballast - soil). Here, the soil is described by a coupled lumped mass (CLM) foundation recently proposed by the authors [13]. This model takes into account the track/foundation interaction without considering a whole soil model requiring an excessive calculation time. From the FIEM model, values of these parameters are obtained through the comparison between the continuous and discrete model overall impedances [13].

The railpad and ballast stiffness coefficients $\left(k_{p}\right.$ and $\left.k_{b}\right)$ as well as the damping coefficients $\left(d_{p}\right.$ and $\left.d_{b}\right)$ are finally obtained by fitting the model with the 


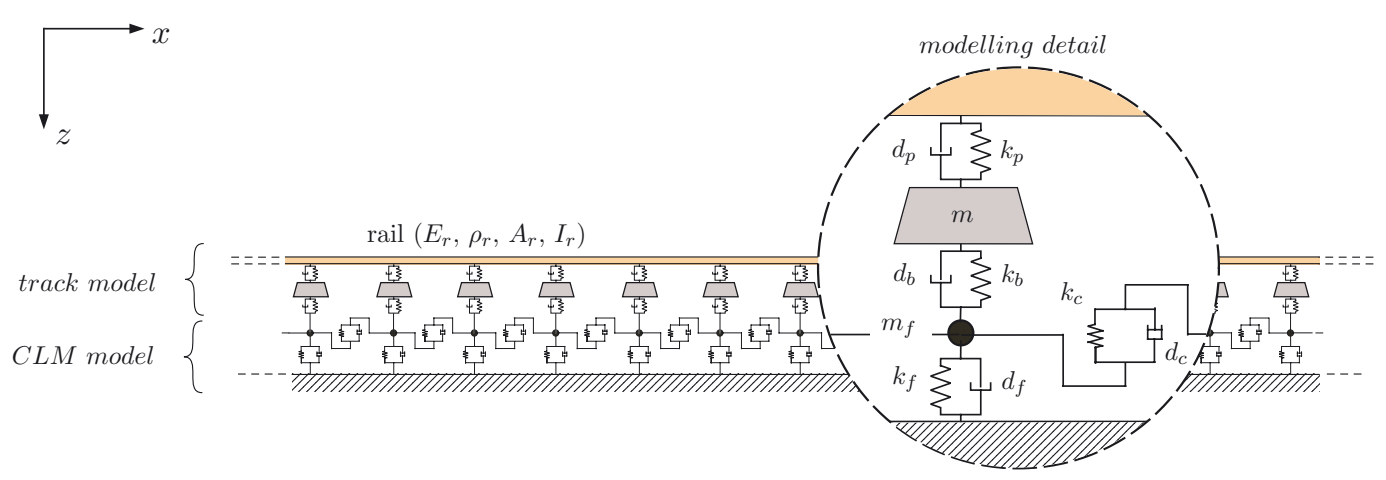

Fig. 5. Flexible track/foundation model used for the parameters fitting.

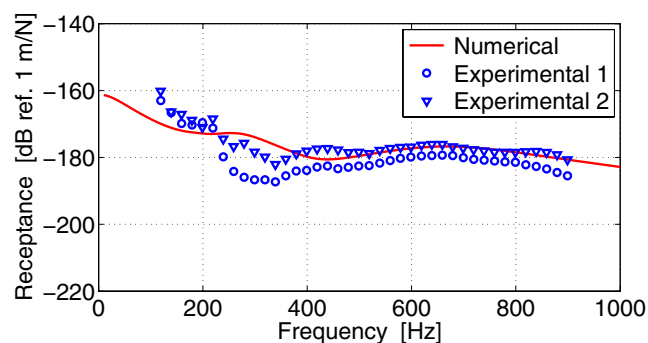

(a) Direct receptance (response on the rail)

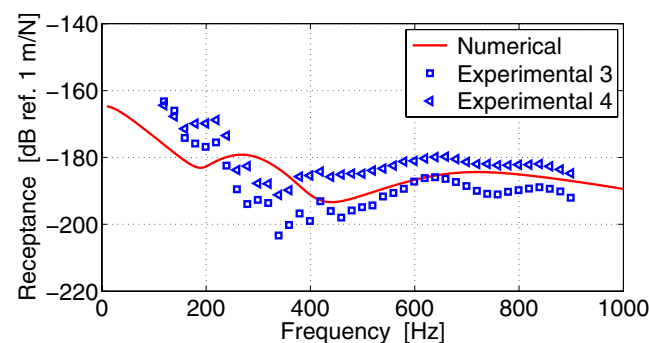

(b) Indirect receptance (response on the rail)

Fig. 6. Updating of the track finite element model with experimental results.

measurements. Figure 6 represents the best fit curves for the vertical receptances of the rail. The updating allows to determine the main resonances of the track vertical motion. The first two modes are respectively at $288 \mathrm{~Hz}$ (the rail and sleepers vibrating in phase) and at $694 \mathrm{~Hz}$ (rail and sleepers vibrating out of phase). The effect of soil flexibility is directly observable on these results with large amplitudes at relatively low frequencies. The third mode, well known as the pinned-pinned mode, is not observed in the rail response since it is located out of the frequency range. It can be therefore estimated at $1430 \mathrm{~Hz}$ by the formula

$$
f_{\mathrm{P}-\mathrm{P}}=\frac{\pi}{2} \sqrt{\frac{E_{r} I_{r}}{m_{r} L^{4}}}
$$

obtained from the Euler-Bernoulli formulation of a simply supported beam (Young's modulus $E_{r}$, length $L$ ). The following values are finally derived for the track:

$$
\begin{aligned}
& k_{p}=550 \mathrm{MN} . \mathrm{m}^{-1} \quad k_{b}=361 \mathrm{MN} . \mathrm{m}^{-1} \\
& d_{p}=68 \mathrm{kNs.m}{ }^{-1} \quad d_{b}=55 \mathrm{kNs.m^{-1 }} .
\end{aligned}
$$

\section{Passage of IC/IR trains and measured soil vibrations in the neighbourhood}

To be concise, only some results are presented in this section. Complete results can be found in our web site where a complementary report can be downloaded. Seismic sensors (Sensor SM-6 low-frequency and Geospace GS-20DM geophones) are used as for the site testing, at various distances $y_{R}$ from the external side of the nearest track (track B, in opposite of track A). Additional geophones, especially dedicated to horizontal motion, have been used to capture the longitudinal and lateral directions, respectively along ( $x$-direction) and perpendicularly ( $y$-direction) to the track. To present concordant results with the soil characteristics, train passing tests were performed the same day for each site.

Measurements in Watermael (site 1). Seventeen passages of domestic trains have been recorded. The particularity of the site 1 of Watermael lies in the existence of a local discontinuity of the track: a rail joint resting upon four wooden sleepers with an irregular spacing. Due to this defect, an important vibration level has been observed.

Measurements in Watermael (site 2). The second site of Watermael is located at a few hundred meters from the first one. There is no local defect and 17 passages have been recorded. Levels are smaller than the ones issued from the previous site, essentially due to the absence of rail joint. Moreover the passing of each train wheelset is less readily identified than for the preceding results. Nevertheless the maximum level is not negligible, presumably explained by the bad quality of the track at this place. 


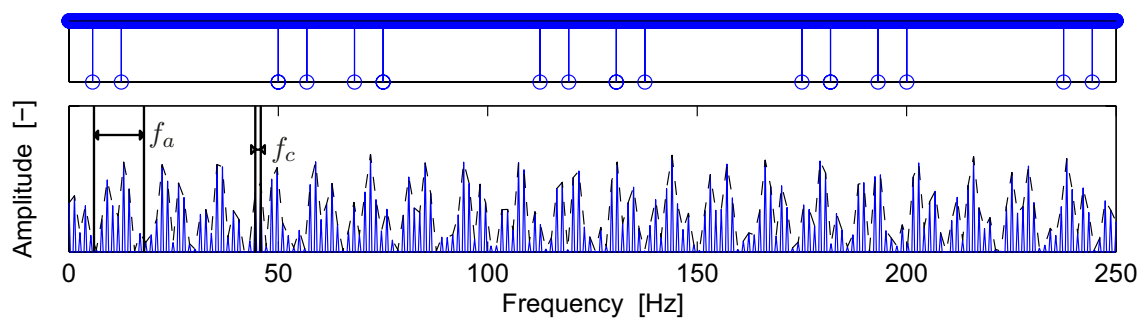

(a) Theoretical excitation mechanisms

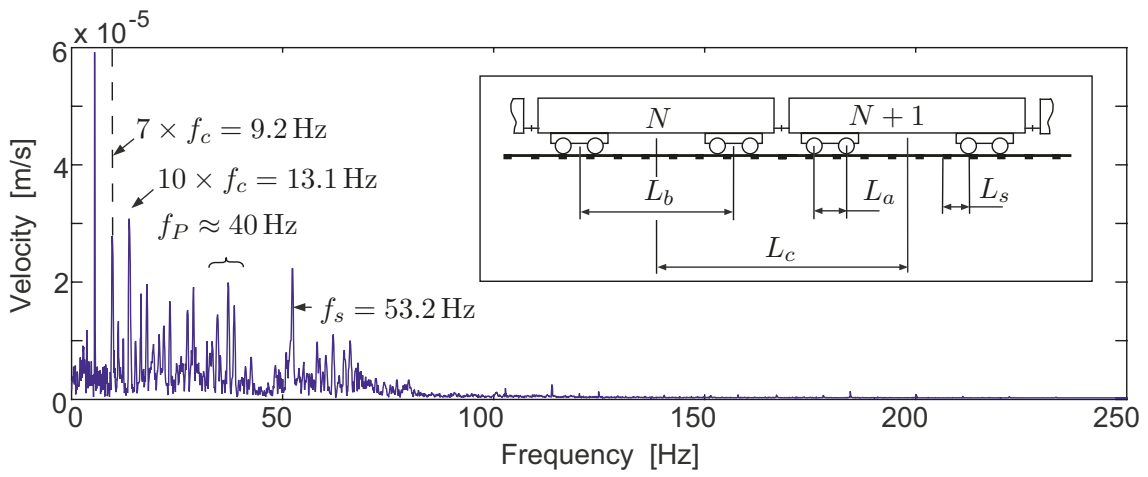

(b) Measured ground vibration at $20 \mathrm{~m}$ from the track

Fig. 7. Spectra content related to the passing of an AM75 at a speed $v_{0}$ of $115 \mathrm{~km} \cdot \mathrm{h}^{-1}\left(L_{\mathrm{c}}=24.4 \mathrm{~m} ; L_{\mathrm{b}}=17.3 \mathrm{~m} ; L_{\mathrm{a}}=2.67 \mathrm{~m}\right.$; $\left.L_{\mathrm{s}}=0.60 \mathrm{~m}\right)$.

Measurements in Ottignies. The last site is located at $25 \mathrm{~km}$ from the previous ones. As for the second site of Watermael, the rail surface does not present any local defect but the soil configuration is different. Two soil layers have been detected, a loam layer with a thickness $h=1.5$ to $2.5 \mathrm{~m}$, lying on a shale halfspace with high rigidity. Conclusions similar to the preceding cases can be drawn.

The interested reader can find the entire results in our web site through a complementary technical report (http://mecara.fpms.ac.be/ georges/ KOU2012-AVE2012_appendix.pdf).

\section{Results and analysis of parameters influencing the level}

Ground vibration is a complex problem that includes several mechanisms in the generation and the propagation of vibration waves. The wheel/axle weight is transmitted to the ground through the sleepers and it is modulated by the vehicle and the track periodicity. In addition to axle loads, the wheel roughness and track irregularity intensifies the ground forces. Some of them can be directly observable in the track deflection. Figure 7 presents the spectral content of a typical result (passage of an AM75 at a speed $v_{0}$ of $115 \mathrm{~km} . \mathrm{h}^{-1}$ ), for which a preliminary analysis can be made. The frequency spectrum shows some peaks (the fundamental and some harmonic frequencies), which can be calculated by

$$
f_{i}=\frac{v_{0}}{L_{i}}
$$

where $L_{i}$ represents a relevant dimension in the vehicle (e.g. the carriage spacing $L_{\mathrm{c}}$ ) or in the track (the sleeper spacing $L_{\mathrm{s}}$ for $f_{\mathrm{s}}$ ). Besides, the soil resonance is pointed up, and is approximately given by

$$
f_{\mathrm{P}}=\frac{c_{\mathrm{P}, 1}}{4 \tilde{h}}
$$

including the compression waves velocity $c_{\mathrm{P}, 1}$ of the first layer and its mean depth $\tilde{h}$. An amplitude modulation is also observable, due to the axle passage frequency $f_{\mathrm{a}}$.

Figure 8 presents an overview of some relevant results in the three sites, based on the peak particle velocity $P P V$ and the maximum of the time-average velocity $M T A V$, calculated by

$$
\begin{aligned}
M T A V & =\max \left(v_{\mathrm{rms}}(t)\right) \\
& =\max \left[\sqrt{\frac{1}{\tau} \int_{0}^{t} v^{2}(\xi) e^{-\frac{t-\xi}{\tau}} \mathrm{d} \xi}\right] \quad(\tau=0.125 \mathrm{~s}) .
\end{aligned}
$$

Some general comments can be made on the collected measurements. Higher levels are generally obtained for the Watermael site 1 , due to the presence of the rail joint. The amplitude decreases strongly with the distance 

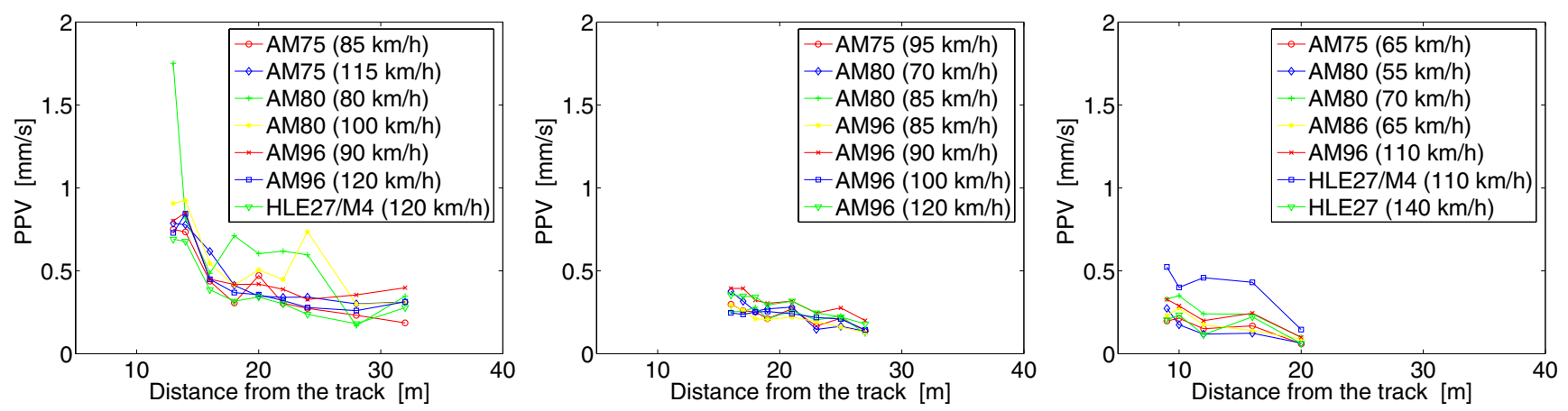

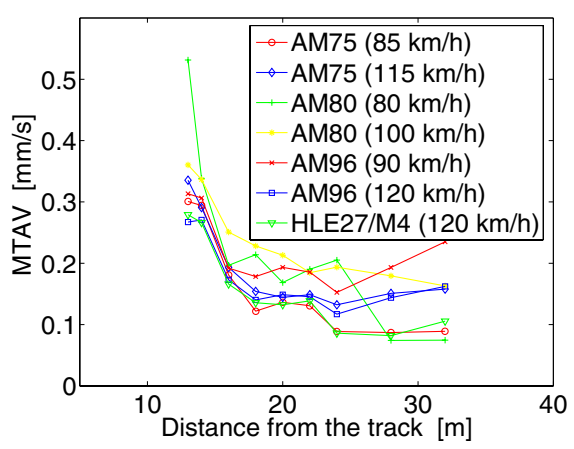

(a) Watermael site 1 - Track B

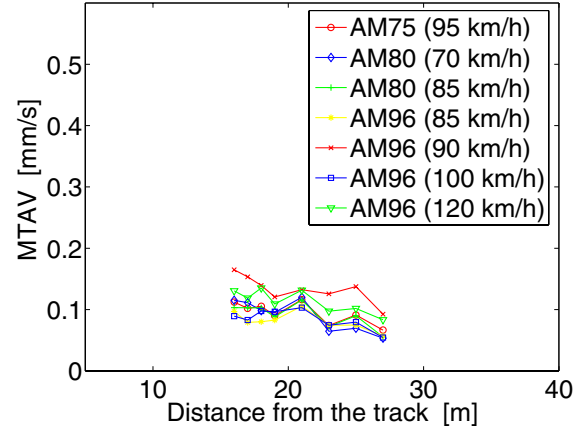

(b) Watermael site 2 - Track B

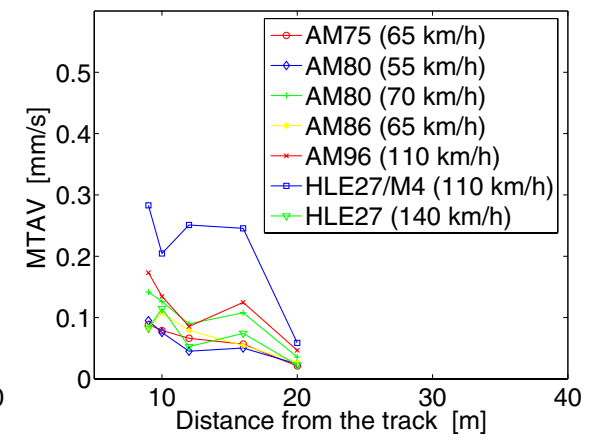

(c) Ottignies - Track A

Fig. 8. Vertical peak particle velocity (top) and maximum time-average velocity (bottom) measured in Watermael site 1 (left), Watermeal site 2 (center) and Ottignies site (right), with respect to the vehicle type

from the track, reaching the value of around $P P V=$ $0.8 \mathrm{~mm} . \mathrm{s}^{-1}$ and $M T A V=0.25 \mathrm{~mm} \cdot \mathrm{s}^{-1}$ for a distance of $15 \mathrm{~m}$. Through all these results for Watermael site 1, it is difficult to emphasize any strong correlation between the level and the train type. For the other sites, no important influence is observed between the different vehicles, apart from vibrations generated by HLE27 locomotives presenting larger levels especially in Ottignies, and, in a lesser extent, by the AM96 units. It is also difficult to establish any tendency with the speed, in the analysed speed range. Horizontal levels cannot be neglected and have the same order of magnitude as ones related to the vertical direction. This observation implies that the evaluation of vibratory nuisance (for human exposure and building damages) has to be done in the three directions.

\section{Conclusion}

An experimental database as complete as possible has been presented, in order to analyse the ground vibrations generated by IC and IR vehicles. These data are interesting, since they are related to domestic trains largely used in Belgium, and complete, with experimental assessment of track/soil configuration. The influence of the site and the track configuration was investigated and some conclusions could be drawn. The paper presents a first step in the evaluation of the railway-induced ground vibrations. The soil configuration and the track dynamics have been analysed so as to obtain relevant parameters to model the track/soil structure. Associated to in situ methods, this approach allows to identify all the dynamic parameters of soils, even for complex geometries. With these informations, the presented data can be used to validate a prediction model for train induced vibrations.

Acknowledgements. The authors wish to acknowledge the Belgian railway engineering and project management office TUC Rail for its support to these experimental investigations.

\section{References}

[1] J. Alias, La Voie Ferrée - Technique de Construction et d'Entretien. 2nd edn., Eyrolles, Paris, 1984

[2] G. Degrande, L. Schillemans, Free field vibrations during the passage of a Thalys high-speed train at variable speed, J. Sound Vib. 247 (2001) 131-144

[3] P. Galvín, J. Domínguez, Experimental and numerical analyses of vibrations induced by high-speed trains on the Córdoba-Málaga line, Soil Dyn. Earthquake Eng. 29 (2009) 641-651

[4] L. Auersch, The excitation of ground vibration by rail traffic: theory of vehicle-track-soil interaction and measurements on high-speed lines, J. Sound Vib. 284 (2005) 103-132

[5] A.M. Kaynia, C. Madshus, P. Zackrisson, Ground vibration from high-speed trains: prediction and countermeasure, J. Geotechnical Geoenviron. Eng. 126 (2000) 531537 
[6] V.V. Krylov, Vibrational impact of high-speed trains, effect of track dynamics, J. Acoust. Soc. Am. 100 (1996) 3121-3134

[7] B. Picoux, R. Rotinat, J.-P. Regoin, D. Le Houédec, Prediction and measurements of vibrations from a railway track lying on a peaty ground, J. Sound Vib. 267 (2003) 575-589

[8] B. Picoux, D. Le Houédec, Diagnosis and prediction of vibration from railway trains, Soil Dyn. Earthquake Eng. 25 (2005) 905-921

[9] G. Kouroussis, O. Verlinden, C. Conti, A two-step time simulation of ground vibrations induced by the railway traffic, J. Mech. Eng. Sci. 226 (2012) 454-472

[10] G. Kouroussis, O. Verlinden, C. Conti, On the interest of integrating vehicle dynamics for the ground propagation of vibrations: the case of urban railway traffic, Vehicle System Dynamics 48 (2010) 1553-1571

[11] G. Kouroussis, O. Verlinden, C. Conti, Free field vibrations caused by high-speed lines: measurement and time domain simulation, Soil Dyn. Earthquake Eng. 31 (2011) 692-707

[12] G. Kouroussis, O. Verlinden, C. Conti, Ground propagation of vibrations from railway vehicles using a finite/infinite-element model of the soil, Proc. IMechE, Part F: J. Rail Rapid Transit 223 (2009) 405-413

[13] G. Kouroussis, G. Gazetas, I. Anastasopoulos, C. Conti, O. Verlinden, Discrete modelling of vertical track-soil coupling for vehicle-track dynamics, Soil Dyn. Earthquake Eng. 31 (2011) 1711-1723 